\title{
¿Qué hicimos bien? Impacto positivo de la pandemia COVID-19 en enfermedades infecciosas en México
}

\author{
What did we do right? Positive impact of the COVID-19 \\ pandemic on infectious diseases in Mexico
}

\author{
Abiel H. Mascareñas de los Santos, ${ }^{*}$ Daniela Cisneros Saldaña ${ }^{\ddagger}$ \\ * Jefe de la División de Infectología Pediátrica. \\ ‡ Médico residente de Infectología Pediátrica, División de Infectología Pediátrica.
}

Departamento de Pediatría del Hospital Universitario «Dr. José Eleuterio González», Universidad Autónoma de Nuevo León. México.

En China, en diciembre de 2019, aparece un brote de neumonía de etiología desconocida, hasta la identificación de un nuevo tipo de coronavirus denominado SARS-CoV-2 por las autoridades sanitarias chinas el 7 de enero de 2020. La enfermedad por Coronavirus 2019, ahora conocida como COVID-19, provocaba un espectro de manifestaciones respiratorias agudas que en algunos pacientes evolucionaba a insuficiencia respiratoria y muerte. Su propagación fuera de ese país fue inminente a pesar de los esfuerzos de mitigación por las autoridades locales, por lo que el 11 de marzo de 2020 fue declarada pandemia por la Organización Mundial de la Salud (OMS). ${ }^{1-4}$

Desde entonces, la enfermedad ha afectado a 192 países con 125'650,992 casos reportados, 2'757,710 muertes en el mundo y 200,211 defunciones en México al día 26 de marzo de 2021. México ocupa el tercer lugar en número de muertes, sólo superado por Estados Unidos de Norteamérica y Brasil, con 546,880 y 303,462 muertes, respectivamente. ${ }^{1-3}$

La pandemia por COVID-19 trajo consigo la implementación de múltiples restricciones sociales con la finalidad de disminuir la transmisión. Dentro de las medidas se estableció el aislamiento de casos sospechosos y sus contactos, cierre de escuelas, trabajo desde casa, campañas de distanciamiento

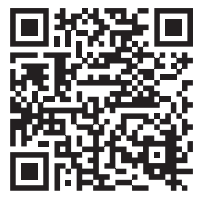

social, uso rutinario de mascarillas, lavado de manos y limpieza de superficies. . $^{1,5}$

En México, se suspendieron las clases en todos los niveles educativos dependientes de la Secretaría de Educación Pública a partir del 23 de marzo de 2020 hasta la fecha, lo que afectó a un estimado de 33.2 millones de niños y adolescentes; medidas que también fueron implementadas por 194 países dentro de los primeras tres semanas de la declaración de pandemia por la OMS. , $^{3,6,7}$

A pesar del sombrío panorama, se han observado beneficios indirectos en la salud pública, debido en parte a que las medidas de distanciamiento social, las campañas nacionales "Quédate en casa» y "Susana distancia», además del lavado de manos, implementadas por autoridades sanitarias con el objetivo de disminuir la transmisión del SARS-CoV-2, probablemente también tuvieron repercusión en otras enfermedades infecciosas, lo cual se pone de manifiesto en los reportes del Sistema Nacional de Vigilancia Epidemiológica, mostrando reducción de más de $50 \%$ en la incidencia de algunas enfermedades infectocontagiosas.

Basados en los reportes del Boletín Epidemiológico del Sistema Nacional de Vigilancia Epidemiológica-Sistema Único de Información de la

Citar como: Mascareñas SAH, Cisneros SD. ¿Qué hicimos bien? Impacto positivo de la pandemia COVID-19 en enfermedades infecciosas en México. Rev Latin Infect Pediatr. 2021; 34 (1): 3-5. https://dx.doi.org/10.35366/99820 
Rev Latin Infect Pediatr. 2021; 34 (1): 3-5

\begin{tabular}{|c|c|c|c|c|c|}
\hline Enfermedad & 2017 & 2018 & 2019 & 2020 & $\begin{array}{c}\text { Reducción respecto } \\
\text { al año previo (\%) }\end{array}$ \\
\hline Coqueluchoide & 2,266 & 2,060 & 3,190 & $873^{*}$ & 72.6 \\
\hline Tos ferina & 622 & 657 & 791 & $233^{*}$ & 70.5 \\
\hline Influenza & 7,280 & 5,951 & 6,963 & $4,800^{* *}$ & 31.1 \\
\hline Parotiditis & 4,585 & 8,773 & 8,009 & $3,494^{\star * *}$ & 56.4 \\
\hline $\begin{array}{l}\text { Enfermedades infecciosas } \\
\text { intestinales }\end{array}$ & 62200,960 & $5^{\prime} 754,640$ & 5788,090 & $2^{\prime} 779,700^{\star * *}$ & 52.0 \\
\hline Hepatitis A & 6,676 & 8,027 & 10,079 & $3,900^{\star \star *}$ & 61.3 \\
\hline Rotavirus & 1,131 & 1,210 & 1,225 & $324^{\star \star \star}$ & 73.6 \\
\hline Fiebre tifoidea & 44,929 & 34,603 & 28,815 & $16,563^{\star \star \star}$ & 42.5 \\
\hline Varicela & 151,609 & 128,903 & 110,986 & $38,108^{\star * *}$ & 65.7 \\
\hline
\end{tabular}

Secretaría de Salud de México, el síndrome coqueluchoide, después de mantenerse entre 2,060 y 3,190 casos en los últimos tres años, en 2020 mostró una reducción de $72.6 \%$ en relación con el año previo, con 873 casos reportados. La tos ferina a su vez, con un rango de 622 y 791 casos anuales, presentó una disminución de $70 \%$, con 233 casos reportados. El número de casos acumulados de influenza en 2020 presentó una reducción de $31.1 \%$ respecto al año previo, con 4,800 casos reportados. - $^{8-10}$

Cabe destacar que la tendencia a la disminución no sólo afectó las enfermedades respiratorias, sino que también se documentó una reducción importante del número de casos en enfermedades gastrointestinales de origen infeccioso, de las cuales una medida fundamental para su prevención es el lavado de manos, implementado de manera sistemática con el uso de soluciones alcoholadas, práctica que se ve reflejada en una disminución de $52 \%$ de infecciones gastrointestinales en 2020 comparada con 2019. El reporte de infecciones por rotavirus presentó una disminución de $73.6 \%$ en el 2020 , destacando también hepatitis A con una reducción de $61.3 \% .^{8-10}$

En la Tabla 1 se muestran algunas enfermedades y el número de casos acumulados anuales, así como la reducción en porcentaje comparando 2019 y 2020.

Una cuestión importante a considerar como sesgo en nuestro análisis es la posibilidad latente de situaciones condicionantes al subdiagnóstico o infrarreporte de enfermedades en el Sistema Nacio- nal de Vigilancia Epidemiológica por varias razones, como la dificultad en la búsqueda de atención médica por parte de la población, en especial si se trata de enfermedades con una historia natural que tienden a la resolución favorable, además del manejo médico de sostén, o la saturación de hospitales y clínicas convertidas en centros de atención COVID-19, limitando la atención de primer contacto, así como la falta de realización de pruebas diagnósticas y su atención médica, y consecuente reporte en el sistema.

Sin duda, en esta temporada invernal fue notoria la marcada disminución y ausencia en algunos centros hospitalarios de los padecimientos usuales en la población pediátrica, como el crup o laringotraqueobronquitis, síndrome coqueluchoide, tos ferina, bronquiolitis y neumonías virales. Haciendo una comparación basada en el Servicio de Pediatría en el Hospital Universitario «Dr. José Eleuterio González» de la Universidad Autónoma de Nuevo León, en el mes de febrero de 2021 se ingresó un paciente con neumonía por COVID-19, y dos más con padecimientos respiratorios, en comparación con 41 casos con enfermedades respiratorias en febrero del 2020, lo que representa una reducción de $92 \%$.

En parte explicado por la sana distancia, limitación de contactos interpersonales con familia en el hogar, y a su vez por el cierre de las escuelas y guarderías, donde la estrecha y prolongada convivencia entre niños actuaba como un eficiente propagador de agentes infecciosos. 
Rev Latin Infect Pediatr. 2021; 34 (1): 3-5

\section{Queda a considerar:}

¿Reemergerán estas enfermedades al volver a la normalidad?, ¿algún patógeno habrá desaparecido de la comunidad?, ¿seguirá siendo el virus sincitial respiratorio el principal agente causal de bronquiolitis?, ¿habrá cambios estacionales en la incidencia de enfermedades respiratorias?, ¿las medidas de sanidad, como el lavado de manos y uso de mascarilla, persistirán?, ¿por cuánto tiempo?, ¿continuaremos viendo los reflejos de estas medidas sanitarias en el futuro?

\section{Reconsiderando, ¿hicimos algo bien?}

\section{REFERENCIAS}

1. Zhang J, Litvinova M, Liang Y, Wang Y, Wang W, Zhao $S$ et al. Changes in contact patterns shape the dynamics of the COVID-19 outbreak in China. Science. 2020; 368 (6498):1481-1486.

2. Johns Hopkins University. COVID-19 Dashboard [Internet]. [citado 26 de marzo de 2021]. Disponible en: https:// coronavirus.jhu.edu/map.html

3. Diario Oficial de la Federación. Acuerdo número 02/03/20 [Internet]. 2020. Disponible en: https://www.dof.gob.mx/ nota_detalle.php?codigo=5589479\&fecha $=16 / 03 / 2020 \#$ : :text=\%2D\%20Se\%20suspenden\%20las\%20clases $\% 20$ del,medio\%20superior\%20y\%20superior\%20dependientes
4. Zheng J. SARS-CoV-2: an emerging coronavirus that causes a global threat. Int J Biol Sci. 2020; 16 (10): 1678-1685.

5. Secretaría de Salud, Gobierno de México. Sana distancia COVID-19 [Internet]. 2020 [citado 7 de marzo de 2021]. Disponible en: https://www.gob.mx/salud/documentos/sanadistancia

6. Gilliam WS, Malik AA, Shafiq M, Klotz M, Reyes C, Humphries JE et al. COVID-19 Transmission in US Child Care Programs. Pediatrics [Internet]. 2021 [citado 7 de marzo de 2021]; 147 (1). Disponible en: https://pediatrics. aappublications.org/content/147/1/e2020031971

7. COVID-19: estudiantes afectados por cierre de escuelas en México [Internet]. Statista [citado 12 de marzo de 2021]. Disponible en: https://es.statista.com/estadisticas/1196749/ estudiantes-afectados-cierre-escuelas-covid-mexico-niveleducativo/

8. Dirección General de Epidemiología. Boletín Epidemiológico. Sistema Nacional de Vigilancia Epidemiológica Sistema Único de Información. Secretaría de Salud; 2018 dic. Report No.: Número 52, Volumen 35 semana 52.

9. Dirección General de Epidemiología. Boletín Epidemiológico. Sistema Nacional de Vigilancia Epidemiológica Sistema Único de Información. Secretaría de Salud; 2019 dic. Report No.: Número 52, Volumen 36 semana 52.

10. Dirección General de Epidemiología. Boletín Epidemiológico. Sistema Nacional de Vigilancia Epidemiológica Sistema Único de Información. Secretaría de Salud.; 2020 dic. Report No.: Número 53, Volumen 37 semana 53.

Correspondencia:

Dr. Abiel H. Mascareñas de los Santos

E-mail: a_mascarenas@hotmail.com 